\title{
Radially restricted linear energy transfer for high-energy protons: a new analytical approach
}

\author{
J. Chen ${ }^{1}$, A.M. Kellerer ${ }^{1,2}$, H.H. Rossi ${ }^{3}$ \\ ${ }^{1}$ GSF-Institute of Radiation Biology, Postfach 1129, D-85758 Oberschleißheim, Germany \\ ${ }^{2}$ Radiobiological Institute, University Munich, D-80336 Munich, Germany \\ ${ }^{3} 105$ Larchdale Ave., Upper Nyack, NY 10960, USA
}

Received: 30 March 1994 /Accepted: 22 April 1994

\begin{abstract}
Radially restricted linear energy transfer (LET) is a basic physical parameter relevant to radiation biology and radiation protection. In this report a convenient method is presented for the analytical computation of this quantity without the need for complicated simulation. The method uses the energy-restricted LET $L_{\Delta}$, as recently redefined in a 1993 ICRU draft document and supplements it by a relatively simple term that represents the energy of fast $\delta$ rays lost within distance $r$ from the track core. The method provides a better fit than other models and is valid over the entire range of radial distance from track center to the maximum radial distance traveled by the most energetic secondary electrons. $L_{r}$ computed by this approach differs only a few percent from the values obtained from explicit Monte Carlo simulations. The concept applies equally to heavy ions and to electrons.
\end{abstract}

\section{Introduction}

Different ionizing particles may have the same linear energy transfer (LET), but depending on velocity and charge, the microdosimetric patterns of energy deposition around their trajectories can be substantially different and can result in different relative biological effectiveness (RBE). Restricted LET can serve as a parameter that accounts for these differences. There are two alternative definitions. Energy-restricted LET is a concept that is most suitable in calculations; radially restricted $L E T$ is more complicated, but also more meaningful in radiobiological considerations, especially when one deals with high-energy heavy ions that are of growing importance with regard to radiotherapy or radiation protection in space. Furthermore, $L_{r}$ is measurable, in contrast to $L_{\lrcorner}$.

Contribution to the international symposium on heavy ions research: space, radiation protection and therapy, 21-24 March 1994, Sophia-Antipolis, France 
The radially restricted LET, $L_{r}$, can be obtained through Monte Carlo computations. However, such computations are complicated and time consuming, and approximations are, therefore, usually employed. Several methods have been chosen in the past that are valid for a limited range of intermediate to large radial distances. In view of the improved definition and the increasing interest in heavy ions, it is desirable to reconsider the existing approximations and to seek an improved treatment that can be used in the entire range of radial distance, from track center to maximal radial distance traveled by the most energetic secondary electrons.

\section{Empirical expressions}

A number of approximative equations for radially restricted LET were developed in the past. They are attractive because of their symplicity of use. Two approaches have been widely used.

Chatterjee et al. (1973) and Chatterjee and Schaefer (1976) presented an equation for the radial energy deposition around the trajectory of a heavy charged particle in water, the core and penumbra model. It is assumed that excitations form a core of high-energy deposition in the center of the particle track, while the $\delta$ electrons transport their energy away from the core and form a penumbra around it. The core radius $r_{c}$ and the penumbra radius $r_{p}$ in microns are set equal to:

$r_{c}=0.0116 \beta$

$r_{p}=0.768 E-1.925 \sqrt{E}+1.257$

where $E$ is the kinetic energy of the particle in megaelectron volts per nucleon, $\beta$ is the speed of the particle in fractions of the speed of light. The formulas apply to water and to particles with kinetic energy larger than $2 \mathrm{MeV} /$ nucleon. Outside the core region the radially restricted LET is given by

$L_{r}=\frac{L_{\infty}}{2}\left[1+\frac{1+2 \ln \left(r / r_{c}\right)}{1+2 \ln \left(r_{p} / r_{c}\right)}\right]$ for $\quad r_{c} \leq r \leq r_{p}$

According to (1), $L_{r}$ increases linearly with $\ln (r)$. As shown in Fig. 1, this model agrees well with simulated results in the penumbra region.

Recently, Xapsos (1992) presented another simple empirical expression for the radially restricted LET:

$L_{r}=\frac{\ln \left\{T_{\max }\left[A\left(2-\Delta / T_{\max }\right)+I\left(1-\Delta / T_{\max }\right)\right] / I^{2}\right\}}{2 \ln \left(T_{\max } / I\right)} \cdot L_{\infty}$

$I$ is the mean excitation energy $\left(I=71.6 \mathrm{eV}\right.$ for water vapor), $T_{\max }$ the maximum energy of secondary electrons, and $\Delta$ the cutoff energy corresponding to the radial distance $r$. The range-energy relation given by Cole (1969) for air and collodion is used to link $\boldsymbol{\Delta}$ (in kiloelectron volts) to $r$ (in microns):

$\Delta=5.9(r+0.007)^{0.565}-0.367$ 


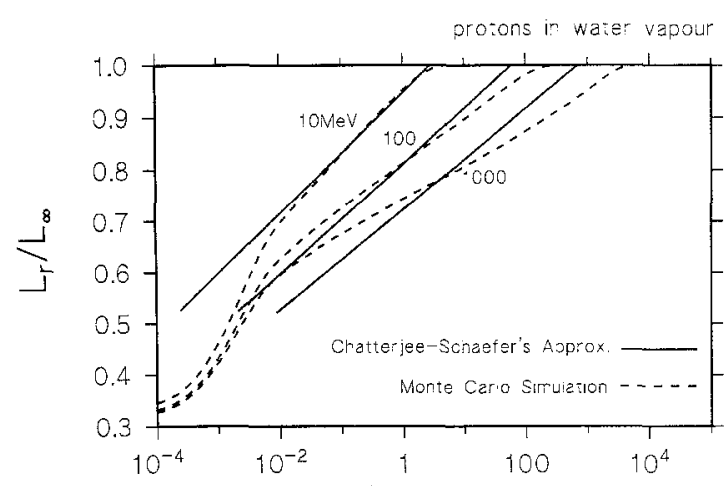

radial distance, $r / \mu m$

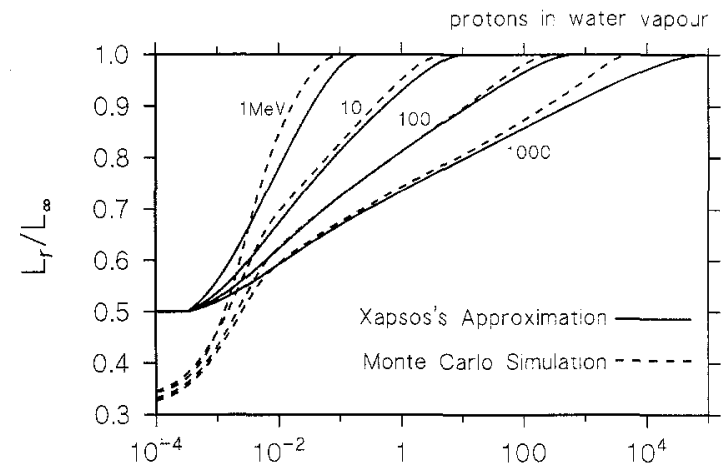

radial distance, $r / \mu m$
Fig. 1. Radially restricted linear energy transfer (LET) in percentage of stopping power as a function of radial distance. Comparison of ChatterjeeSchaefer's approximation with Monte Carlo simulations

Fig. 2. Radially restricted LET in percentage of stopping power as a function of radial distance.

Comparison of Xapsos's approximation with Monte Carlo simulations

As shown in Fig. 2, the values $L_{r}$ obtained by Xapsos's expression agree fairly well with simulations for radial distances in excess of a few nanometers. For small radial distances, $L_{r}$ goes to $0.5 L_{\infty}$, which is too high.

Empirical models are simple and convenient to use, if the relevant parameters, such as core and penumbra radii or corresponding range-energy relation, are known. Their validity is, however, restricted to relatively large radial distances.

\section{Analytical approach}

An alternative to the empirical fits to $L_{r}$ has been chosen by Baum (1969). This approach links $L_{r}$ to the energy-restricted LET by adding to it a suitable correction term. In his formulation Baum had to refer to $L_{A}$ in its conventional definition. Considering a cylinder of radius $r$ that corresponds to the range traveled by an electron with kinetic energy of $\Delta$, he noted that $L_{r} \geq L_{\Delta}$, the missing term being the energy deposited in the cylinder by $\delta$ electrons with kinetic energy larger than $\Delta$. To account for this energy and to account, further- 


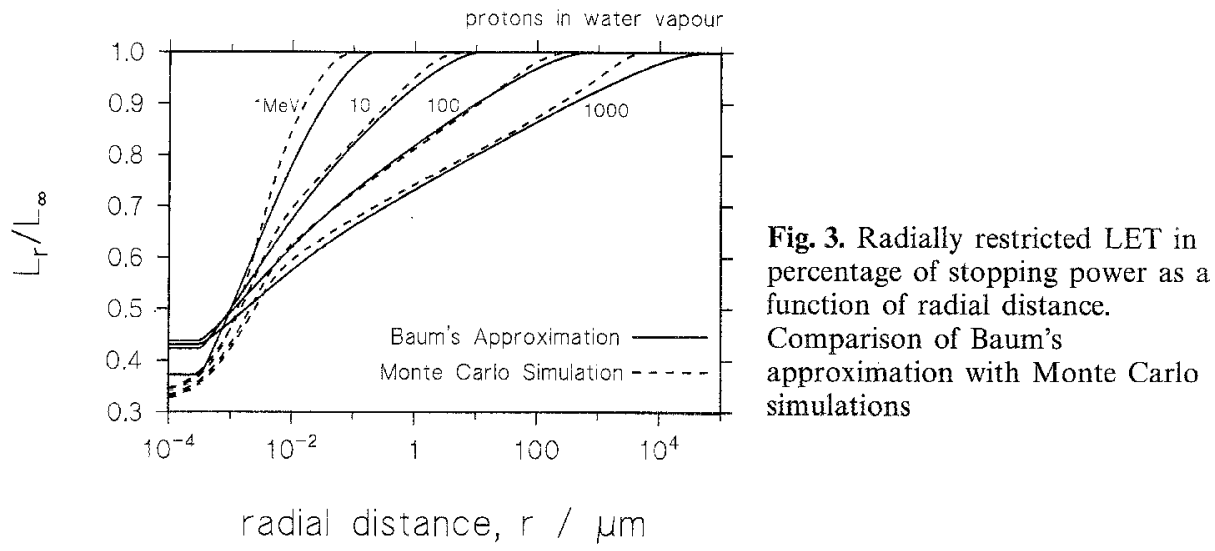

more, for the inadequacy of the former definition of $L_{A}$, he used the formula

$L_{r}=L_{\Delta+13.5}+\frac{\mathrm{d} N}{\mathrm{~d} x}(\Delta+13.5)$

The value $13.5 \mathrm{eV}$ is the mean ionization energy in producing secondary electrons. The term $\mathrm{d} N / \mathrm{d} x$ is the number of collisions with energy loss of the particles greater than $A+13.5$, which can be calculated from the Bohr equation for heavy ions. $L_{\Delta+13.5}$ is the energy-restricted LET, which is to be calculated from the Bethe formula. The range-energy relation, which links radial distance $r$ to the cutoff energy $\Delta$ is, as in the formulation by Xapos, taken from the equation given by Cole. For protons with different kinetic energies, comparisons of (3) with the results of Monte Carlo simulations are shown in Fig. 3. For radial distances in excess of a few nanometers Baum's model agrees fairly well with the simulations.

The approach chosen by Baum is sufficiently attractive to serve as the basis of a further improvement which makes use of the new definition of energyrestricted LET in the 1993 ICRU draft document (Allisy et al. 1993).

In its new definition the energy-restricted LET contains the binding energy for secondary electrons with kinetic energies in excess of cutoff energy 4 :

$L_{\Delta}=\frac{\rho N_{A} Z}{M_{A}} \sum_{i}\left(\int_{0}^{\Delta} \sigma_{i}(T, E)\left(T+I_{i}\right) \mathrm{d} T+\int_{\Delta}^{T_{\max }} \sigma_{i}(T, E) I_{i} \mathrm{~d} T\right)$

and it can, therefore, replace the approximation $L_{\Delta+13.5}$ that is used by Baum. When the redefined $L_{A}$ is used, the missing term for calculation of $L_{r}$ results only from the energy of fast $\delta$ rays lost within distance $r$ from the track core. If $\boldsymbol{\Delta}$ is the energy of the electrons with range $r$, the simplest approximation for this missing term is that all $\delta$ electrons with energy larger than $\Delta$ deposit a part of their kinetic energy within radius $r$ that equals the given cutoff energy $\Delta$. With this approximation one obtains an expression for $L_{r}$ :

$L_{r}=L_{\Delta}+\frac{\rho N_{A} Z}{M_{A}} \Delta \sum_{i} \int_{\Delta(r)}^{T_{\text {max }}} \sigma_{i}(T, E) \mathrm{d} T$ 


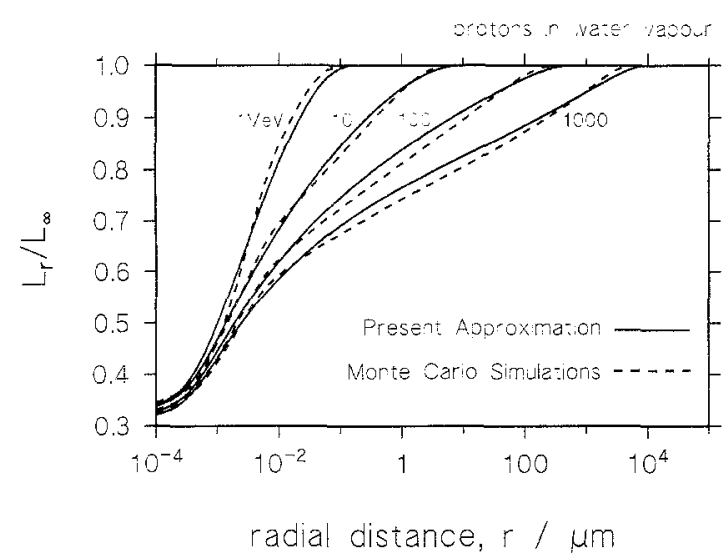

Fig. 4. Radially restricted LET in percentage of stopping power as a function of radial distance.

Comparison of present approximation with Monte Carlo simulations

where $\sigma(T, E)$ is the differential ionization cross section. The relationship between $r$ and $\Delta$ is given by an equation for electrons in water from ICRU Report 16 (1970):

$$
\Delta=\exp \left[1.8488+0.567173 \ln r-0.019612(\ln r)^{2}+0.002836(\ln r)^{3}\right]
$$

The results of (5) are given in Fig. 4. Comparing the results with Monte Carlo simulations, one sees that in the entire radial distance range (5) gives a good approximation for the radially restricted LET.

\section{Discussion}

Radially restricted LET is a basic physical parameter relevant ro radiation biology and radiation protection. In our study a convenient method is presented for the analytical computation of this quantity. The method is analogous to the earlier approach by Baum (1969). It improves this approach by utilizing the new definition of the energy-restricted LET $L_{\Delta}$. The radially restricted LET calculated with this method agrees well with the result of a Monte Carlo simulation, the differences being at most a few percent. A comparison with experimental results is not made here, as there is sufficient evidence in the literature to show that the results of the empirical expression, as well as Monte Carlo simulations, are in accordance with experimental results for larger radial distances. At small radial distances near the track center, however, experimental results are uncertain. Those uncertainties arise mainly from the fact that the experiments did not measure the energy deposition directly, but electric current or light emission produced by secondary electrons. At small radial distances, especially for distances smaller than $1 \mathrm{~nm}$, the Monte Carlo simulations give, therefore, more reliable results. The results of Monte Carlo simulations presented here agree well with the results given by Hamm with OREC-Code (Waligorski et al. 1986).

The analytical approach is given in terms of interaction cross sections. Using the redefined energy-restricted LET, one needs ionization cross sections as they are given in the literature in analytical form (Rudd 1990) for the calculation 
of $L_{r}$. The method is of general validity and applies equally for heavy ions and electrons. Since radially restricted LET is an integrated form of radial dose distribution, the formula can also be utilized to derive the radial dose distribution around the particle trajectories.

\section{Appendix: cross sections used in the computations}

\section{Protons}

For proton energies considered here, the main interactions are impact ionizations and excitations. In ionization processes the semiempirical differential ionization cross sections for proton scattering in water vapor are used that have been derived by Rudd (1990). These cross sections cover the energy range up to $5 \mathrm{MeV}$. To extend their applicability to higher energies, the total ionization cross sections of Rudd are extrapolated by the non-relativistic asymptotic Bethe formula given by Inokuti (1971) for proton energies up to $20 \mathrm{MeV}$ and are further extrapolated by the relativistic asymptotic Bethe formula for proton energies greater than $20 \mathrm{MeV}$. The corresponding differential ionization cross sections were then renormalized by these asymptotic expressions.

Excitation processes were represented by cross sections for electrons of the same velocity. The excitation cross sections for electron transport in water vapor are taken from a Monte Carlo program written by Zaider et al. (1983). The same extrapolation methods as described above are also used for the excitation cross sections. This treatment of excitation processes of protons appears to be admissible, since the values obtained with this data set for the total stopping power in water are in good agreement with the values given in the literature (ICRU Report 49, 1993).

\section{Electrons}

The physical data for the interaction processes, elastic scattering, excitations and ionizations were given in the appendix of a previous paper (Kellerer et al. 1992). We were encouraged to use this set of cross sections for calculations of electron transport in water vapor, since the cross sections reproduce the collision stopping power (ICRU Report 37, 1984) with good precision.

\section{References}

Allisy A, Jennings WA, Kellerer AM, Müller JW, Rossi HH (1993) Radiation quantities and units, interaction coefficients - dosimetry. ICRU News December

Baum W (1969) Comparison of distance- and energy-restricted linear energy transfer for heavy particles with 0.25 to $1000 \mathrm{MeV} / \mathrm{amu}$. In Proceedings of the 2nd Symposium on Microdosimetry, edited by H.G. Ebert, Stresa (Italy). Italy, 653-664

Chatterjee A, Maccabee HD, Tobias CA (1973) Radial cutoff LET and radial cutoff dose calculations for heavy charged particles in water. Radiat Res 54:479-494

Chatterjee A, Schaefer HJ (1976) Microdosimetric structure of heavy ion tracks in tissue. Radiat Environ Biophys 13:215-227

Cole A (1969) Absorption of $20 \mathrm{eV}$ to $50000 \mathrm{eV}$ electron beams in air and plastic. Radiat Res 38:7-33

ICRU Report 16 (1970) Linear energy transfer. International Commission on Radiation Units and Measurements, Washington D.C. USA

ICRU Report 37 (1984) Stopping powers for electrons and positrons. International Commission on Radiation Units and Measurements, Bethesda, USA

ICRU Report 49 (1993) Stopping powers and ranges for protons and alpha particles. International Commission on Radiation Units and Measurements, Bethesda, USA 
Inokuti M (1971) Inelastic collisions of fast charged particles with atoms and molecules the Bethe Theory Revisited. Rev Mod Phys 43:297-347

Kellerer AM, Hahn K, Rossi HH (1992) Intermediate dosimetric quantities. Radiat Res 130:1525

Rudd M (1990) Cross sections for production of secondary electrons by charged particles. Radiat Prot Dosim 31:17-22

Waligorski MPR, Hamm RN, Katz R (1986) The radial distribution of dose around the path of a heavy ion in liquid water. Nucl Tracks Radiat Meas 11:309-319

Xapsos MA (1992) A spatially restricted linear energy transfer equation. Radiat Res 132:282287

Zaider M, Brenner D, Wilson WE (1983) The applications of track calculations to radiobiology I. Monte Carlo simulation of proton tracks. Radiat Res 95:231-247 